\title{
Efficient Nonlinear Activation Function in Optical Neural Network
}

\author{
H. Zhang ${ }^{1}$, L. X. Wan ${ }^{1}$, M. Gu ${ }^{2,3}$, X. D. Jiang ${ }^{1 \dagger}$, J. Thompson ${ }^{3}$, H. Cai ${ }^{4}$, S. Paesani ${ }^{5}$, R. Santagati ${ }^{5}$, A. Laing ${ }^{5}$, G. Q. Lo ${ }^{6}$, D. \\ L. Kwong ${ }^{4}$, L. C. Kwek ${ }^{1,3+}$, and A. Q. Liu ${ }^{1 \dagger}$ \\ ${ }^{1}$ Quantum Science and Engineering Centre (QSec), Nanyang Technological University, 50 Nanyang Ave, 639798, Singapore \\ ${ }^{2}$ Complexity Institute and School of Physical and Mathematical Sciences, Nanyang Technological University, 639798, Singapore \\ ${ }^{3}$ Centre for Quantum Technologies, National University of Singapore, Block S15, 3 Science Drive 2, 117543, Singapore \\ ${ }^{4}$ Institute of Microelectronics, A*STAR (Agency for Science, Technology and Research), 138634, Singapore \\ ${ }^{5}$ Centre for Quantum Photonics, H. H. Wills Physics Laboratory and Department of Electrical and Electronic Engineering, University of Bristol, \\ Merchant Venturers Building, Woodland Road, Bristol BS8 1UB, UK \\ ${ }^{6}$ Advanced Micro Foundry, 11 Science Park Road, 117685 Singapore \\ *Corresponding Authors: exdjiang@ntu.edu.sg, cqtklc@nus.edu.sg, eaqliu@ntu.edu.sg
}

\begin{abstract}
An efficient nonlinear activation function for optical neural network is proposed and demonstrated by executing complex-valued algorithms and intensity measurement. Great learning capability are achieved by the inherent properties of the complex-valued optical neural network.

OCIS codes: (250.5300) Photonic integrated circuits; (100.4997) Pattern recognition, neural networks
\end{abstract}

\section{Introduction}

Appropriate selection of nonlinear activation function in optical implementation of neural networks[1] are vital as without a nonlinear activation function, the neural network can only solve linearly separable problems. Great interests are raised in proposing the optical nonlinear activation functions[2][3]. Here in our work, we propose and demonstrate an optical neural network executing complex-valued neural network. Efficient and inherent nonlinear activation are achieved by the optical waves themselves together with intensity measurement. Unlike previous proposals of optical nonlinear activation functions, no additional optical devices are required for realizing those activation function designed for electronic devices. The optical neural network itself can shape diverse nonlinear decision surfaces. Great advantages are proved in our implementations regarding the learning capability and convergence, which makes optical implementations of neural network competitive to electronic implementing.

\section{Design and theoretical Analysis}

The nonlinearity of a neural network can be revealed directly from its decision surface. Here we take a two-input and linearly inseparable dataset, which is usually called as the half-moon dataset as an example. By a real-valued $2 \times 2$ layer, the output is $\left[\begin{array}{l}y_{1} \\ y_{2}\end{array}\right]=\left[\begin{array}{ll}w_{11} & w_{12} \\ w_{21} & w_{22}\end{array}\right]\left[\begin{array}{l}x_{1} \\ x_{2}\end{array}\right]+\left[\begin{array}{l}b_{1} \\ b_{2}\end{array}\right]$. The decision surface is shaped by solving the equation $y_{1}=y_{2}$ and thus a straight line $l_{1}:\left(w_{11}-w_{21}\right) x_{1}+\left(w_{12}-w_{22}\right) x_{2}+\left(b_{1}-b_{2}\right)=0$. In optical neural networks, while intensity measurement extends the decision boundary by $\left|y_{1}\right|=\left|y_{2}\right|$, resulting in two straight lines $l_{1}$ and $l_{2}$ : $\left(w_{11}+\right.$ $\left.w_{21}\right) x_{1}+\left(w_{12}+w_{22}\right) x_{2}+\left(b_{1}+b_{2}\right)=0$. By complex-valued weight matrices where $w_{j k}=p_{j k}+i q_{j k}$ and $b_{j}=$ $b_{R j}+i b_{I j}$, together with intensity measurement, interactions can be introduced into the weight elements and thus nonlinear decision surface are shaped by

$$
\sqrt{\left(p_{11} x_{1}+p_{12} x_{2}+b_{R 1}\right)^{2}+\left(q_{11} x_{1}+q_{12} x_{2}+b_{I 1}\right)^{2}}=\sqrt{\left(p_{21} x_{1}+p_{22} x_{2}+b_{R 2}\right)^{2}+\left(q_{21} x_{1}+q_{22} x_{2}+b_{I 2}\right)^{2}}
$$

Composition of nonlinear surfaces by linear mappings after measurement strengthen the learning capability of a single layer of optical neural network. The up-mentioned decision surfaces are as shown in Fig 1.

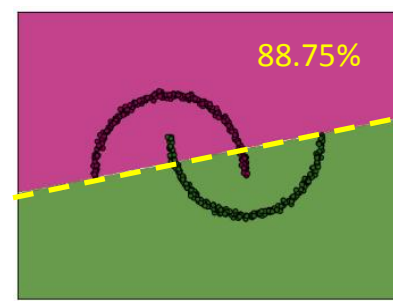

(a)

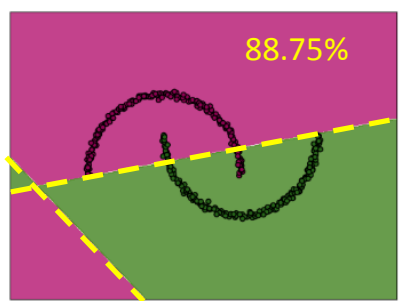

(b)

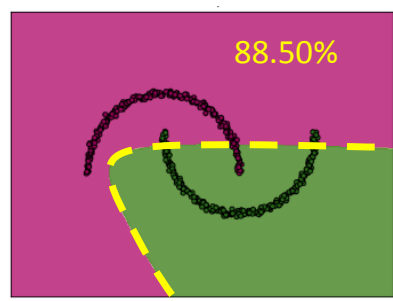

(c)

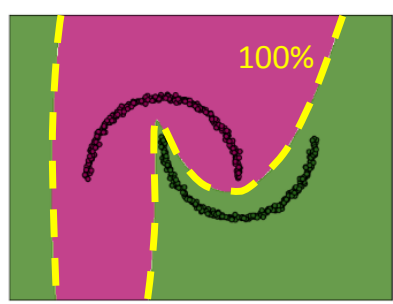

(d)

Fig. 1. Decision surfaces of (a) real-valued layer (b) real-valued optical layer with intensity measurement (c) complex-valued optical layer and (d) complex-valued optical layer with linear mappings.

\section{Experimental setup}

The chip in testing an 8-mode linear optical circuit following Reck's scheme. It fulfills the functionality of input preparation, arbitrary complex-valued weight matrices (singular-vector-decomposition). Intensity measurement are performed the output ports of the chip. The diagram is as shown in Fig 2. 


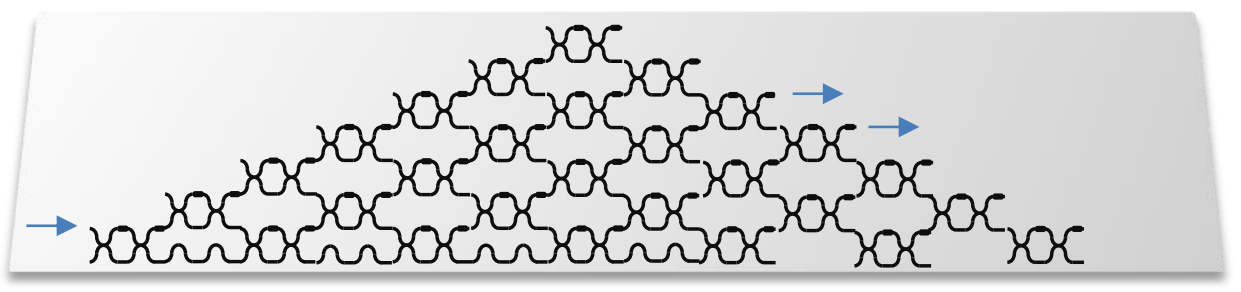

Fig. 2. Experimental setup.

\section{Results and Discussions}

We test the chip on three tasks. The first two tasks are classification of toy datasets, the circle and the half-moon. We implement the input $x_{1}, x_{2} \in[-1,1]$ stepwise onto the chip and perform intensity measurement at the two output ports of the chip. The recorded light intensities are as shown upside in Fig $\mathbf{2 a}$ and $\mathbf{2 b}$. The resulting decision surface are shown accordingly. The resolution of the decision surface are determined by the step size of input. Another task is 4input XOR problem. A classification accuracy of $100 \%$ are achieved with a single layer of optical neural network. The recorded light intensity is shown in Fig 3c.
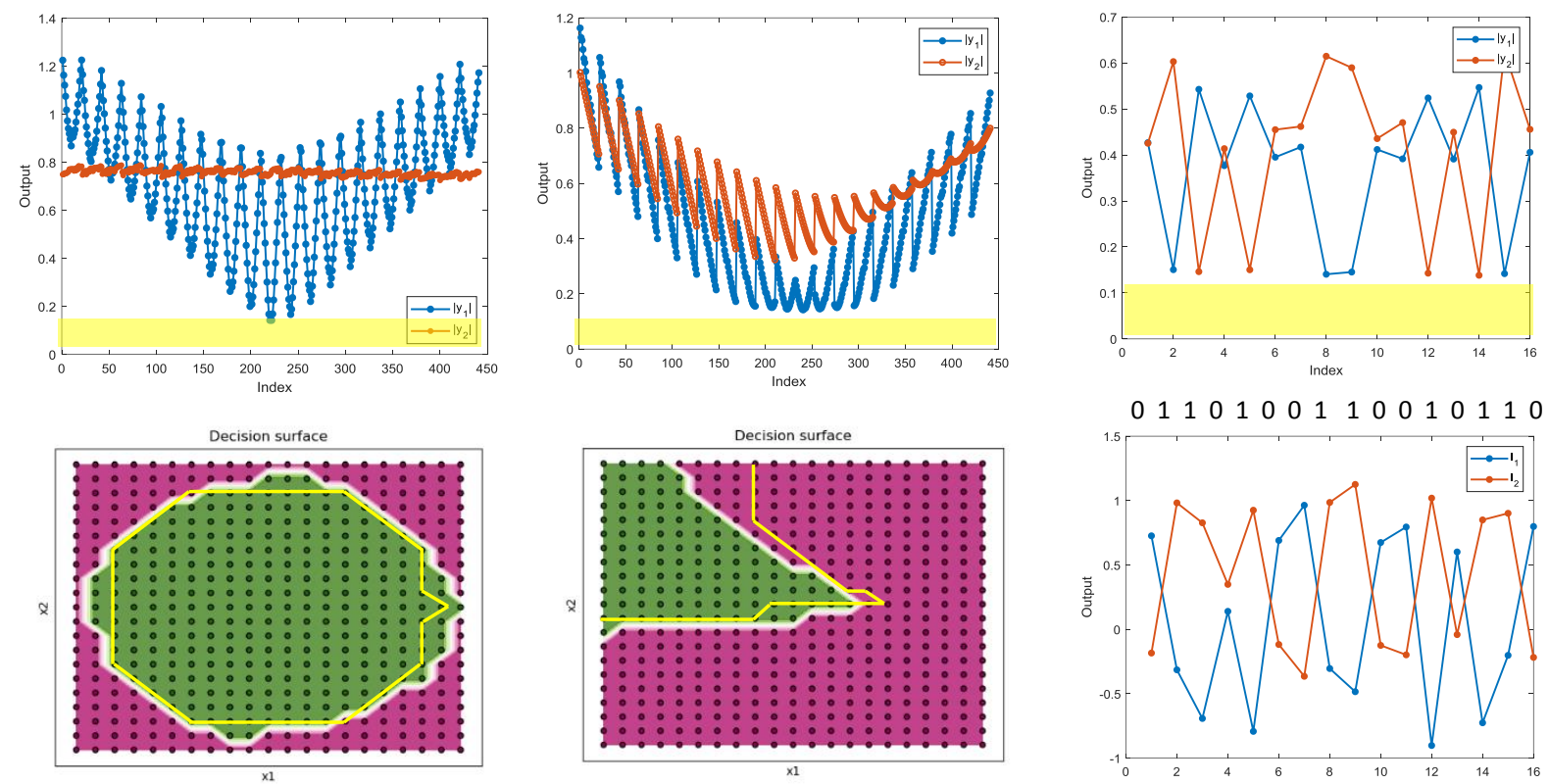

(a)

(b)

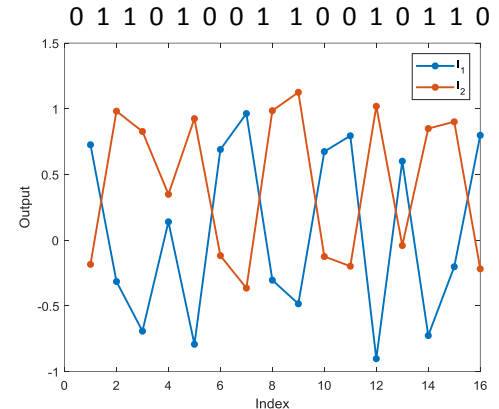

(c)

Fig. 3. Experimental results of (a) classification of the circle dataset and (b) the half-moon dataset and (c) the four-input XOR problem.

\section{Conclusions}

An efficient nonlinear activation function for optical neural networks are proposed by executing the complex-valued neural networks and intensity measurement. The nonlinearity is created by the inherent property of the optical network itself with no additional cost and optical loss. The created nonlinear activation is demonstrated to work in between different layers and thus promising for demonstrating all-optical neural networks. The performance of complex-valued neural networks with such nonlinear activations outperforms conventional real-valued networks with functions like Relu.

\section{Acknowledgement}

This work was supported by the Singapore Ministry of Education (MOE) Tier 3 grant (MOE2017-T3-1-001).

\section{References}

[1] Shen, Yichen, et al. "Deep learning with coherent nanophotonic circuits." Nature Photonics 11.7 (2017): 441.

[2] Williamson, Ian AD, et al. "Reprogrammable Electro-Optic Nonlinear Activation Functions for Optical Neural Networks." arXiv:1903.04579 (2019).

[3] Zuo, Ying, et al. "All Optical Neural Network with Nonlinear Activation Functions." arXiv preprint arXiv:1904.10819 (2019). 